\title{
3D Shape Acquisition Using an FTP-based Method in Product Modeling
}

\author{
Chunsheng $\mathrm{Yu}$ \\ Department of Mechanical \\ and Manufacturing \\ Engineering \\ University of Manitoba, \\ Winnipeg, Manitoba, \\ R3T 5V6, Canada \\ umyuc1@cc.umanitoba.ca
}

\author{
Lushen $\mathrm{Wu}$ \\ Department of Mechanical \\ and Electronic Engineering, \\ Nanchang University, \\ Jiangxi, \\ 330029, China \\ lushenwu@hotmail.com
}

\author{
Qingjin Peng* \\ Department of Mechanical \\ and Manufacturing \\ Engineering \\ University of Manitoba, \\ Winnipeg, Manitoba, \\ R3T 5V6, Canada \\ pengq@cc.umanitoba.ca
}

\begin{abstract}
Three-dimensional (3D) shape modeling is one of the most fundamental processes in CAD/CAM systems. There is a variety of methods to build $3 D$ shapes for product design and manufacturing. The methods include defining a $3 D$ object using solid or feature modeling methods, or building a $3 D$ shape of the existing object using reverse engineering techniques. Image-based shape recovery techniques provide effective tools in reverse engineering to acquire $3 D$ data of objects.

This paper reports a simple method to reconstruct a $3 D$ object from its $2 D$ (two-dimensional) image for the product modeling. A method based on FTP (Fourier Transform Profilometry) phase analysis is proposed to measure the $3 D$ surface of an object. The comparison of the FTP method with other methods is discussed and the process of the FTP method is provided. The experiment shows the accuracy and speed of the method.
\end{abstract}

Keywords: Reverse engineering, $3 D$ modeling, CAD/CAM, Image processing

\section{Introduction}

CAD/CAM (Computer Aided Design and Manufacturing) systems are widely used to improve the quality and efficiency of product design and manufacturing. 3D (Three-dimensional) geometric modeling is the most fundamental processing in any CAD/CAM systems. A 3D model can be built in $\mathrm{CAD} / \mathrm{CAM}$ systems using solid or feature modeling

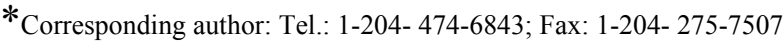

methods, but building an existing object using current $\mathrm{CAD} / \mathrm{CAM}$ systems is time-consuming [1]. An efficient 3D modeling method is needed.

One alternative of 3D modeling methods is using a tracking system. The tracking system can build a 3D model by positioning a probe on the object and trigger the computer to record 3D coordinates of positions of the probe. The obtained 3D coordinates can be used to build the 3D model. Coordinate Measuring Machine $(\mathrm{CMM})$ is a robust 3D mechanical tracker. Electromagnetic and ultrasonic trackers are also used in some tracking systems. These tracking systems are limited by mechanical or electromagnetic problems in the object size, measurement volume and material used [2].

Industrial X-Ray Computed Tomography (CT) is also an approach to build 3D models [3]. It scans an object using X-Ray through the object and gets a series of slices of the object. The slices can be used to produce 3D models of the object. But the system is generally very expensive and needs a big space for installation.

The laser has been an accurate tool for building 3D models of an existing object. There are four methods that use laser to acquire 3D object data [4]. The first is Time/Light in Flight. It is based on the direct measurement of the time of flight of a laser pulse. A laser pulse is reflected back to the receiving sensor by the object and a reference laser pulse is passed through an optical fiber and received by the sensor, the time difference between the two pulses can be converted to distance. The second method is the point laser triangulation. The principle of the point laser triangulation is given in Figure 1 [5]. A laser beam is focused on the object surface producing the light spot $\mathrm{S}$. 
The spot $\mathrm{S}$ is focused at $\mathrm{S}^{\prime}$ on the image plane. The height between the spot $\mathrm{S}$ and a reference plane is $\mathrm{h}$. The angle between laser beam and the lens center $O$ is $\theta$. The distance between the spot $S^{\prime}$ and the reference plane is $\mathrm{dx}$ on the image plane. From the geometry, $h=d x / \sin (\theta)$. The third method is the laser speckle pattern sectioning. Laser radar 3D imaging, also known as speckle pattern sampling, is a 2D (two-dimensional) slice of object's 3D Fourier transform. Two or more 2D slices of object's 3D transform are taken using a CCD (charged couple device) array at each different laser wavelength, and the individual frames are added up to generate a 3D data array. A 3D Fourier transform is applied on this data array to obtain the 3D shape of an object. The fourth method is the laser tracking system. Differing from mechanical, electromagnetic, ultrasonic trackers, the laser tracker uses an interferometer to measure distance, and two high accuracy angle encoders determine vertical and horizontal angles. However, the processing is slow using a laser beam to capture 3D data because the surface has to be scanned line-by-line. The system is expensive and the highenergy laser beam needs to be treated with care [2].

This research searches for a novel solution for the 3D shape capture in product design and manufacturing. The simple structure and cost-effective technique are the objectives of the research.

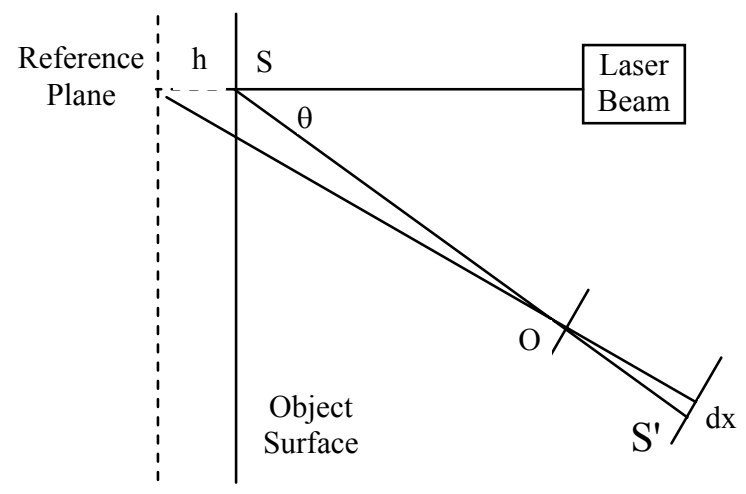

Figure 1. The principle of laser triangulation

Comparing with the $\mathrm{CAD}$ package, tracking systems, industrial CT or laser scanners, the imagebased $3 \mathrm{D}$ reconstruction techniques provide effective tools to acquire 3D data of objects. The FTP (Fourier Transform Profilometry) is proposed as the method to reconstruct the 3D shape of an object. Details will be discussed in following parts of the paper. The accuracy and system requirement of the method are also analyzed when a low-cost hardware (a common projector and digital camera) is adopted. Experimental examples show the performance of the method.
This paper is organized as follows: Section 2 is the literature review of image-based 3D shape reconstructions. In Section 3, the algorithms of FTP and four-step PMP method are introduced. The FTP method is analyzed in Section 4. The process of FTPbased 3D shape acquisition is discussed in Section 5. Finally Section 6 gives the experimental result of the FTP method.

\section{Literature Review of Image-based 3D Shape Reconstructions}

\subsection{Shape-from-shading (SFS)}

SFS is a method that can reconstruct the 3D shape of an object by the mapping between the shading and surface shape in terms of the reflectance function $I(x, y)=R(p, q)$, where $I(x, y)$ denotes image intensity, $p=z x$ and $q=z y, z$ is the depth of the object and $(\mathrm{x}, \mathrm{y})$ are projected spatial coordinates of the 3D object [6]. SFS methods can be divided into four groups: minimization approaches, propagation approaches, local approaches, and linear approaches [7].

The photometric stereo method is the multi-image version of SFS. Rather than using a single intensity image in the conventional SFS, the photometric stereo method uses two or more intensity images of the object under different illumination conditions, which improves the accuracy of the result.

In SFS method, the reflection model of an object's surface has to be assumed. However, real images of object's surface do not always follow the assumed model. Therefore SFS method is somewhat inaccurate and is sensitive to noise.

\subsection{Moiré methods}

In Moiré methods there are two gratings, one is a master grating and the other is a reference grating, from which contour fringes can be generated and resolved by a CCD camera. Using the contour fringes the 3D shape of an object can be obtained [4]. Moiré methods have phase discrimination problems when the surface is not smooth. This problem makes Moiré methods cannot avoid errors when the slope of the surface is greater than a limitation. The system structure and algorithm of Moiré methods is complex.

\subsection{Photogrammetry}

In photogrammetry methods, two images have to be acquired by either two cameras at the same time or by one camera at different time. Then image coordinates of any feature points in two images can be measured. Using the image coordinates of these points, the 3D coordinates of the feature points can be calculated, and 
the whole 3D object can be reconstructed. This method can be used for multiple images. There are two photogrammetry methods: (1) Calibration-based Model construction [8, 9], and (2) Calibration-free Model construction $[10,11]$

The key to these methods is the correspondence problem that is to determine the point in one image that corresponds to a give point in the other image. Generally, it is hard to solve the correspondence problem on a smooth surface. It is necessary to project some additional signs onto the object surface to find correspondence points on all captured images. So far, there are many strategies to project different structured lighting patterns onto the object to recover $3 \mathrm{D}$ shape of an object [12]. The purpose of these strategies is to improve the accuracy of recovery and increase flexibility of different techniques. These strategies have provided good solutions for the 3D shape acquisition, but some drawbacks related these techniques include: complicated patterns and algorithms in the processing of captured images, and expensive to form an application [2].

\subsection{Phase-measuring profilometry (PMP)}

In this method, a fringe pattern is projected onto the object by varying the phase of the pattern. Three or more deformed fringe pattern images are captured by a CCD camera and the phase distribution of the object can be calculated by these images. The $3 \mathrm{D}$ shape of the object can be recovered by mapping the phase distribution to the height [13]. Many effective phasemeasuring algorithms have been established [14].

\subsection{Fourier transformation profilometry (FTP) [15]}

In FTP, a Ronchi grating or a sinusoidal grating is projected onto the object surface. The deformed grating image is captured by a CCD camera. The $3 \mathrm{D}$ shape of the object can be obtained by calculating Fourier transformation of the image, filtering in spatial frequency domain and calculating inverse Fourier transformation. FTP is a widely used method, because it needs only one (or two) image(s) to do full field analysis and the result is accurate.

Comparing with SFS, FTP is accurate and is robust to noise. Comparing with the Moire method, FTP can accomplish fully automatic distinction between a depression and an evaluation of the object shape. It requires no fringe order assignments or fringe center determination, and it requires no interpolation between fringes because it gives height distribution at every pixel over the entire fields. Comparing with the photogrammetry method, there are no complicated patterns and correspondence points to be calculated. The comparison between FTP and PMP will be discussed in the following part. Therefore the FTP is selected to construct 3D shape of objects from 2D images in this research.

\section{The algorithm of FTP and four-step PMP method}

\subsection{The relationship between object's 3D shape and phase}

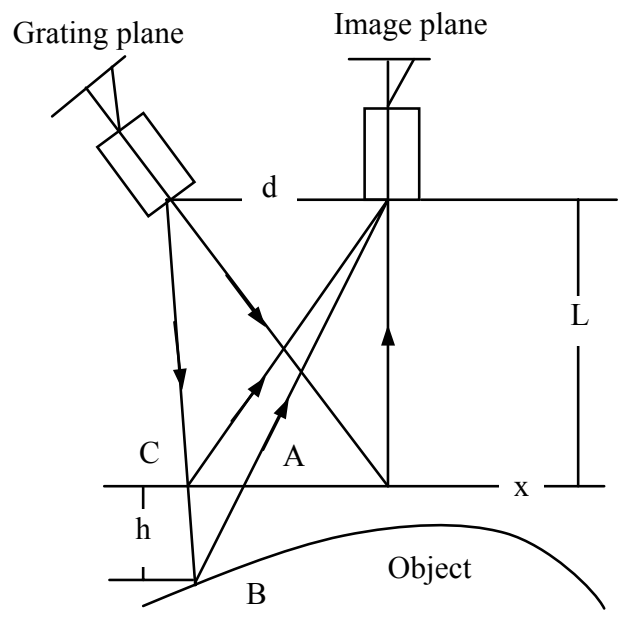

Figure 2. The geometry of the projection and imaging system.

Figure 2 shows the geometry of the projection and imaging system. Point $\mathrm{P}$ and $\mathrm{E}$ are the centers of the exit pupils for projection and the imaging system, respectively. The phase and height relationship is obtained based on the Ref. [16]. Thus,

$h(x, y)=\frac{L}{d} A C=\frac{L}{d} \frac{\varphi_{C D}}{2 \pi f}=k \varphi_{C D}$

where $\mathrm{L}$ is the distance between the image plane and the reference plane; $d$ is the distance between center of the image and center of the projector; $f$ is the spatial frequency of the projected fringes in the reference plane and $k=L /(2 d \pi f)$ is the coefficient, which is related to the configuration of the optical measuring system; $\varphi_{C D}$ is the phase, which contains the surface height information.

If the phase $\varphi_{C D}$ is obtained then the height information of the surface can be calculated. FTP and PMP are methods to compute the phase $\varphi_{C D}$.

\subsection{FTP method}

As shown in Figure 2, when the object is put on the reference plane, the deformed fringe pattern observed on the image plane can be expressed by 
$g(x, y)=r(x, y) \sum_{n=-\infty}^{n=\infty} A_{n} \exp \left(i\left(2 \pi n f_{0} x+n \varphi(x, y)\right)\right)$

when $h(x, y)=0$, the deformed grating image is written as

$g_{0}(x, y)=r_{0}(x, y) \sum_{n=-\infty}^{n=\infty} A_{n} \exp \left\{\left(2 \pi n f_{0} x+n \varphi_{0}(x, y)\right)\right)$

where $r(x, y)$ and $r_{0}(x, y)$ are non-uniform distributions of reflectivity on the surface of the object and on the reference plane, respectively. $A_{n}$ is the weighting factors of Fourier series, $f_{0}$ is the fundamental frequency of the observed grating image, $\varphi(x, y)$ is the phase resulting from the object height distribution and $\varphi_{0}(x, y)$ is the original phase when $(h(x, y)=0)$.

The 1D (one dimension) Fourier transform of the observed image (in Equation (2)) is computed and the Fourier spectrum of the image is obtained. A filter function is used to obtain the fundamental component of the Fourier spectra. The inverse Fourier transform is applied to the fundamental component, an image that only carries the deformed grating information is obtained:

$\hat{g}(x, y)=A_{1} r(x, y) \exp \left(i 2 \pi f_{0} x+\phi(x, y)\right)$

The same operation is applied to Equation (3), then

$\hat{g}_{0}(x, y)=A_{1} r_{0}(x, y) \exp \left(i 2 \pi f_{0} x+\phi_{0}(x, y)\right)$

The phase changed $\Delta \varphi(x, y)$ that has relationship with the height distribution is:

$\Delta \varphi(x, y)=\operatorname{Im}\left\{\log \left[\hat{g}(x, y) \hat{g}_{0}{ }^{*}(x, y)\right]\right\}$

\subsection{Four-step PMP}

In this paper, four-step PMP is used to compare with FTP method. In this method, four phase-shifted sinusoidal fringe patterns are projected onto the object surface with phase shift of $0, \pi / 2, \pi$ and $3 \pi / 2$ within one period of the fringe. The following equations are four intensity distributions on images with the phase shift:

$$
\begin{aligned}
& I_{1}(x, y)=a(x, y)+b(x, y) \cos \varphi(x, y) \\
& I_{2}(x, y)=a(x, y)+b(x, y) \cos [\pi / 2+\varphi(x, y)] \\
& I_{3}(x, y)=a(x, y)+b(x, y) \cos [\pi+\varphi(x, y)] \\
& I_{4}(x, y)=a(x, y)+b(x, y) \cos [3 \pi / 2+\varphi(x, y)]
\end{aligned}
$$

where $a(x, y)$ is the average intensity (background), $b(x, y)$ is the intensity modulation, and $\varphi(x, y)$ is the phase to be determined. The phase $\varphi(x, y)$ can be obtained as follows:

$$
\varphi(x, y)=\arctan \frac{I_{4}(x, y)-I_{2}(x, y)}{I_{1}(x, y)-I_{3}(x, y)}
$$

\subsection{Phase unwrapping}

Since the phase calculated by FTP or PMP gives principal values ranging from $-\pi$ to $\pi$. The phase distribution is wrapped into this range and has discontinuities with $2 \pi$ jumps when the phase variation is larger than $2 \pi$. These discontinuities can be corrected by adding or subtracting $2 \pi$ according to the phase jump ranging from $-\pi$ to $\pi$ or vice versa.

\section{The analysis of proposed methods}

In this section, the comparison of the FTP method and the four-step PMP is discussed based on the experimental result. Two phase-unwrapping methods are used in our experiments, the result of two phaseunwrapping methods is discussed.

\subsection{Experimental setup}

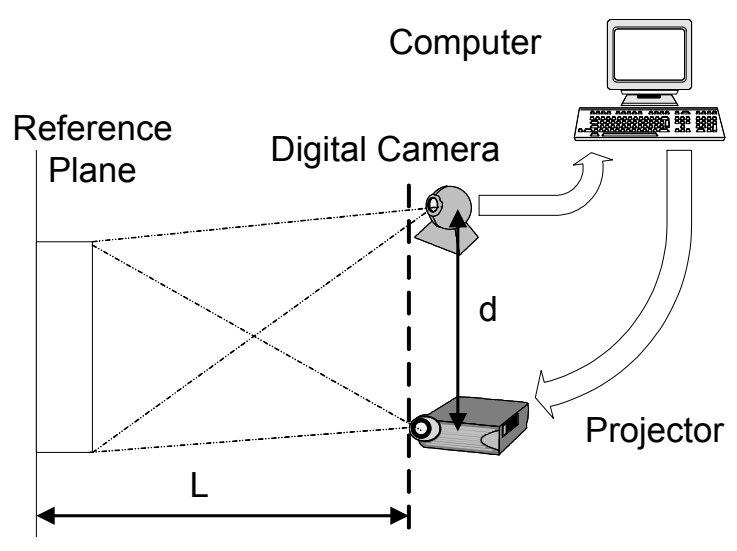

Figure 3. Experimental setup

Figure 3 shows the system layout of FTP and PMP methods. According to the projection geometry of Figure 2, centres of the projection and the CCD camera are located at the same distance $\mathrm{L}$ from the reference plane, and $d$ is the distance between them. A sinusoidal grating is generated by the computer and is projected on the object and the reference plane by the projector. It allows users to do the phase shifting for PMP method and to change the cycle time of the grating for FTP method at a low cost. Images of the deformed grating and the object are captured by the digital camera and saved in the computer. The projector used is an EIKI LC-7000 projector. The resolution of the projector is $1024 \times 768$ pixels. The digital camera is SONY FDMavica. The resolution of the camera is $1024 \times 768$ pixels. FTP and PMP algorithms are developed using MATLAB. 


\subsection{Comparison of FTP and four-step PMP}

Experiments aimed at the comparison of FTP and four-step PMP. Because some errors could be brought into the system during building the system, a computer-generated model was used to test algorithms of FTP and four-step PMP methods. There is no errors brought by the hardware using the computer-generated model, therefore a computer-generated model was used to test algorithms of FTP and four-step PMP methods.

(1) The simulation test

The computer-generated model is a semi-sphere. To simulate the projection of a sinusoidal grating on the semi-sphere, the deformed grating image can be computed by Equation (12) [16].

$I(x, y)=A+B \cos \left[2 \pi f_{0} x+\varphi(x, y)+\alpha_{i}\right]$

where $I(x, y)$ is the gray intensity of pixel $(\mathrm{x}, \mathrm{y})$ of the deformed grating image. $\mathrm{A}$ is the average intensity (background), $\mathrm{B}$ is the intensity modulation, $f_{0}$ is the fundamental frequency of the grating image, $\alpha_{i}$ is the angle of phase shifting, and $\varphi(x, y)$ is the phase to be determined. The phase $\varphi(x, y)$ can be obtained as follows:

$\Delta \phi(x, y) \mathrm{M}-\frac{2 \pi f_{0} d}{L} h(x, y)$

where $h(x, y)=\sqrt{R^{2}-x^{2}-y^{2}}$ and $\mathrm{R}$ is the radius of the semi-sphere. $\mathrm{L}$ is the distance between the image plane and the reference plane; $d$ is the distance between center of the image and center of the projector.

In the test, parameters were set as $\mathrm{A}=100$ candela, $\mathrm{B}=80$ candela, $\mathrm{d} / \mathrm{L}=0.1$ and $\mathrm{R}=40 \mathrm{~mm}$. The sinusoidal grating image was set to 5 pixels per cycle. The $\alpha_{i}$ was set to 0 in FTP method. Figure 4 shows the result of the FTP method. The $\alpha_{i}$ was set to $0, \frac{\pi}{2}, \pi, \frac{3 \pi}{2}$ in fourstep PMP method. Figure 5 shows the result of PMP method. The results of the simulation test show that the 3D model of the semi-sphere is reconstructed correctly by two algorithms (as shown in Figure 4 (c) and Figure 5(c)) therefore it proves that the algorithms are correct.

(2) Experiments and analysis

A pot was used as an example as shown in Figure 6. Figure 6(a) is the original image of the pot, (b) is a 3D plot of the pot that is reconstructed by FTP method, and (c) is a $3 \mathrm{D}$ plot of the pot that is reconstructed by four-step PMP method. In Figure 6 (b), the shape of the pot is smooth and the features of the pot are reconstructed. But in Figure 6 (c), the shape of the pot is rough and with a lot of noise on the surface. The results show that the shape of the pot is reconstructed by FTP method is more accurate than that recovered by four-step PMP method. The reason is discussed as follows.

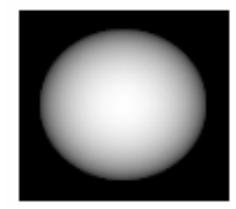

(a)

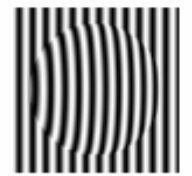

(b)

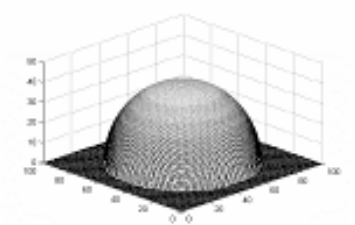

(c)

Figure 4. (a) Image of the semi-sphere without grating; (b) Image of the semi-sphere with deformed grating; (c) 3D plot of the reconstructed semi-sphere.

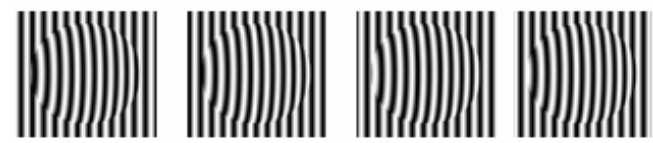

(a)

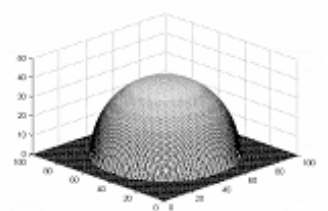

(b)

Figure 5. (a) Four phase-shifted deformed grating image; (b) 3D plot of the reconstructed semi-sphere.

Figure 7 (a) is the 1D spatial frequency spectra of simulation images (Figure 6(b)). Figure 7 (b) is the 1D spatial frequency spectra of deformed grating images (Figure 6(a)). Q0 is the spectrum of background of the images, Q1 is the spectrum of the fundamental component (the grating frequency carrier), and Q2 is the spectrum of all frequency that is higher than the fundamental component. In Figure 7 (a), there are no system errors in the simulation image therefore there is only Q1 in the spatial frequency spectra. In Figure 7(b), there are some signals that were brought in by the experiment system and environments therefore there are Q0 and Q2 in the spatial frequency spectra except Q1. Four-step PMP method can reconstruct the semisphere using simulation image but it fails in reconstructing the pot. The reason is that all image 
information is used in 3D object reconstruction. If the system errors (Q0 and Q2) are bigger than the fundamental component (Q1) the method will fail to reconstruct the object. FTP method uses a filter to remove the $\mathrm{Q} 0$ and $\mathrm{Q} 2$ before the $3 \mathrm{D}$ object reconstruction therefore it can reconstruct the object in a noised environment. Therefore the FTP method has a better anti-noise function than four-step PMP method.

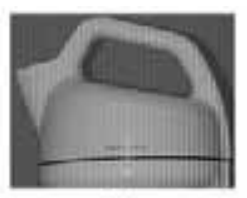

(a)

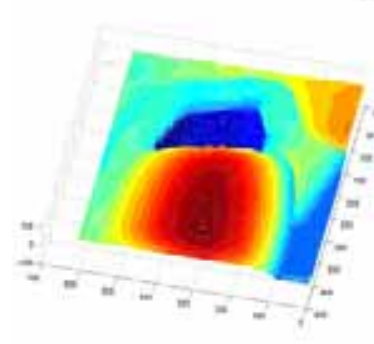

(b)

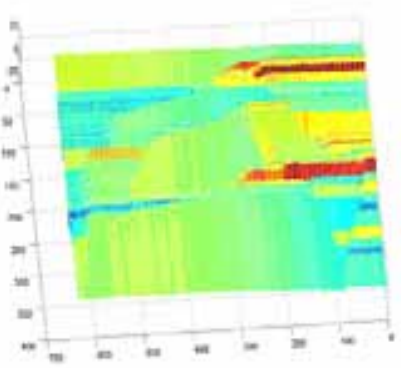

(c)
Figure 6. (a) The original image of the pot; (b) 3D plot of the pot reconstructed by FTP method; (c) 3D plot of the pot reconstructed by Four-step PMP method.

\subsection{Phase unwrapping algorithms}

Noises can be brought in by projectors, shadows or irregular surface brightness. To improve the accuracy of the system, a robust phase unwrapping algorithm is necessary. A common phase unwrapping algorithm and the reliability-guided phase unwrapping algorithm were compared in this research. The common phase unwrapping algorithm scans the wrapped phase image (the wrapped phase image can be obtained by FTP or four-step PMP method) from the top to bottom line by line. For each line, the phase jump between adjacent pixels is checked. If the phase jump is greater than $\pi$ or less than $-\pi$ then $2 K \pi$ is added to the phase of the pixel. $\mathrm{K}$ is added by 1 when there is a phase jump. The reliability-guided phase unwrapping algorithm computes the modulation $M(x, y)$ of the deformed grating image [17]. The phase unwrapping starts from the pixel with maximum modulation and unwraps the phase along a path from the pixel with a higher modulation to the pixel with a low modulation. The advantage of this approach is that it can always unwrap phase in local minimum areas. Figure 8 shows the comparison of the common phase unwrapping algorithm and the reliability-guided phase unwrapping algorithm. More accurate result is obtained by using reliability-guided phase unwrapping algorithm.

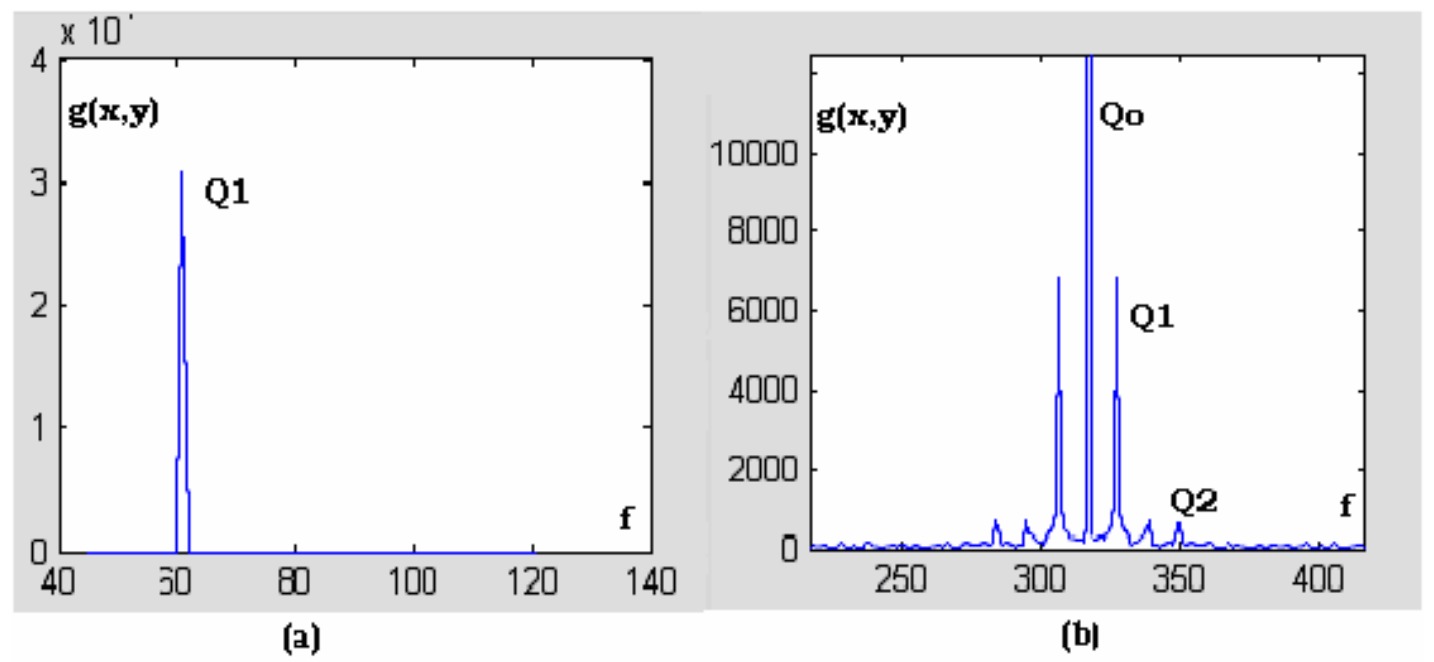

Figure 7. (a) The 1D spatial frequency spectra of simulation images;

(b) The 1D spatial frequency spectra of deformed grating images. 


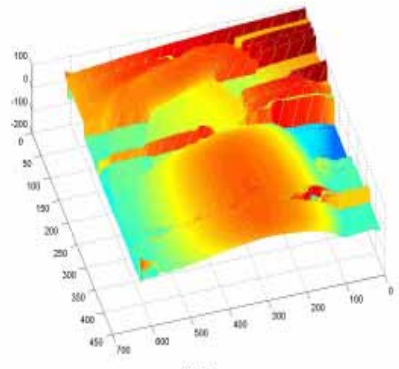

(a)

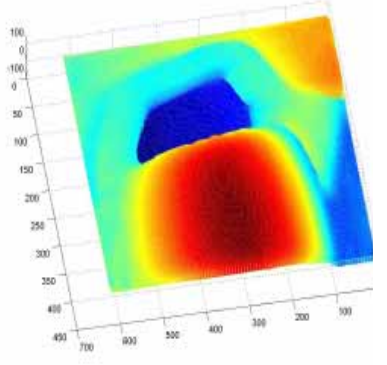

(b)
Figure 8. (a) 3D pot model that is generated by common phase unwrapping algorithm; (b) 3D pot model that is generated by reliability-guided phase unwrapping algorithm.

\section{The process of FTP based 3D shape acquisition}

The overall structure of the FTP method is shown in Figure 9. The process of the FTP method is described as follows.

(1) System calibration is the procedure to accurately determine parameters in the 3D shape acquisition system. The parameters are geometrical parameters of the system and internal parameters of the digital camera. The geometrical parameters are the $\mathrm{L}$ and $\mathrm{d}$ of the system. It can be obtained using a calibration object and the detail can be found in Ref. [18]. The internal parameters of the digital camera are: focal length, principal point, and skew coefficient, distortions. They can be obtained by the software named Camera Calibration Toolbox in Matlab [19].

(2) Sinusoidal grating is generated by Equation (14).

$$
I(x, y)=A+B \cos \left[2 \pi f_{0} x+\alpha_{i}\right]
$$

where $I(x, y)$ is the gray intensity of pixel $(\mathrm{x}, \mathrm{y})$ of the sinusoidal grating image. $\mathrm{A}$ is the average intensity (background), $\mathrm{B}$ is the intensity modulation, $f_{0}$ is the fundamental frequency of the grating image, $\alpha_{i}$ is the angle of phase shift.

(3) Based on Equation 6, two images are required. One is the reference-grating image, the other is deformed grating image.

(4) Grating image pro-processing is for removing the noise in acquired images. Low-pass filter of image processing is used in this procedure.

(5) In phase calculation, images are computed by 1D Fourier transform to obtain the Fourier spectra. Then the spectra are filtered to obtain the fundamental component with a mid-pass filter function. Finally, the inverse Fourier transform is applied to the fundamental component to obtain $g(x, y)$ and $g_{0}(x, y)$, the phase can be obtained using Equation 6.

(6) In phase unwrapping, the reliability-guided phase unwrapping is used to obtain the continuous phase distribution.

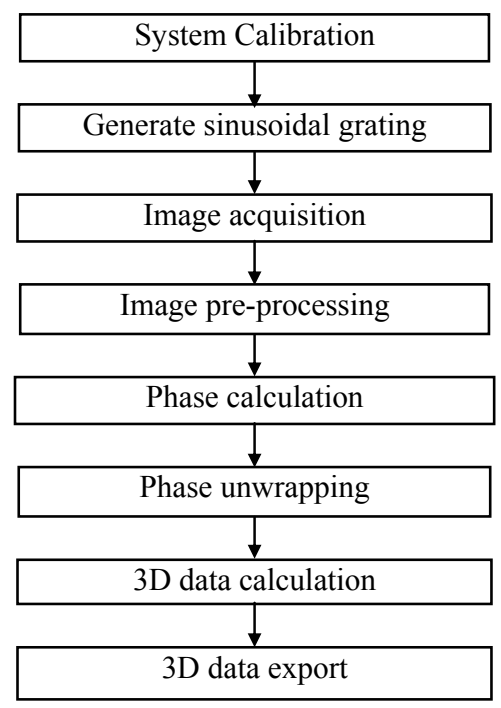

Figure 9. The overall structure of the FTP algorithm

(7) Based on the phase information, the 3D coordinates of data points on the object can be calculated. As shown in Figure 10, the world coordinate system is set that $\mathrm{X}, \mathrm{Y}$ coordinates are on the reference plane and the coordinate centre is in a line with the coordinate centre of the camera coordinate system. $\mathrm{X}, \mathrm{Y}$ and $\mathrm{Z}$ are parallel with the $\mathrm{x}, \mathrm{y}$ and $\mathrm{z}$ of the camera coordinate system. The principal point of the camera is assumed at the centre of the image, the skew coefficient and distortions are zero. The focal length is obtained in the camera calibration. Therefore the $3 \mathrm{D}$ coordinates of a data point on the object can be calculated as below:

$\mathrm{Z}$ coordinate is the height $(\mathrm{h}(\mathrm{x}, \mathrm{y}))$ from a point on the object to the reference plane and it can be calculated by Equation (1).

X coordinate: $X=\frac{U *(\text { focus }+L)}{\text { focus }}$

where $U$ is the coordinate of a pixel in the image coordinate system, focus is the focus length of the camera and can be obtained from camera calibration, and $\mathrm{L}$ is the distance from the image plane to the reference plane.

Y coordinate: $Y=\frac{U^{*}(\text { focus }+L)}{\text { focus }}$ 
Where $U$ is the coordinate of a pixel in image coordinate system.

(8) The 3D data of the object obtained by Equation (7) are the point cloud, they can be output to $\mathrm{CAD} / \mathrm{CAM}$ systems to build the 3D model.

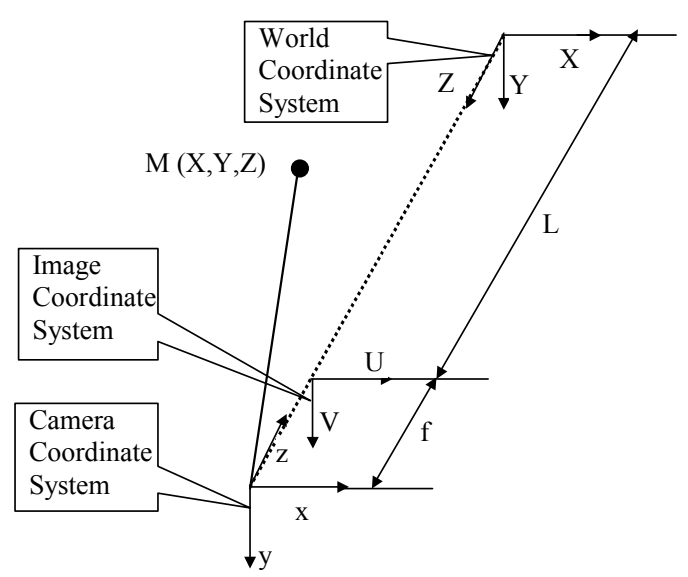

Figure 10. Coordinate system of the FTP method

\section{Experiments and Results}

The experiment is set up as shown in Figure 3. A holder is used as an example in the experiment. Figure 11 is an original image of the holder. After the system calibration, parameters of the system are obtained. $\mathrm{L}=1300 \mathrm{~mm}, \mathrm{~d}=200 \mathrm{~mm}$ and focus $=51.81 \mathrm{~mm}$. Figure 12 is the sinusoidal grating image of 8 pixels per cycle and the size is $1024 \times 768$ pixels.

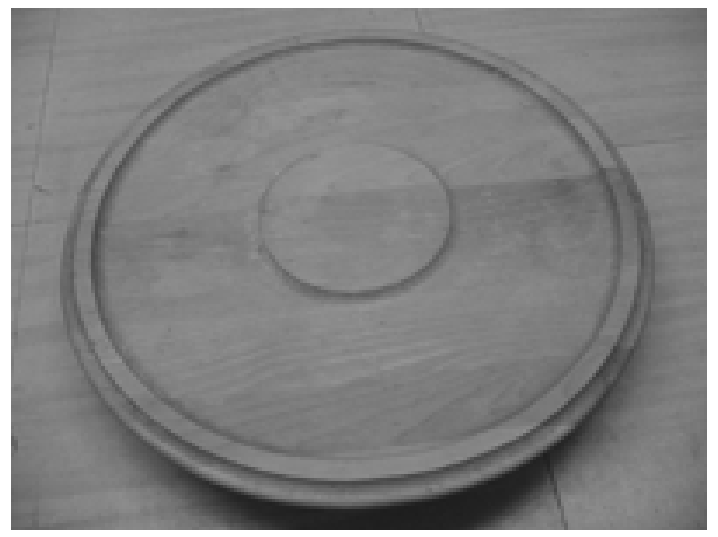

Figure 11. Original picture of the holder

Figure 12 shows a picture of a deformed grating image, and the straight grating lines in Figure 13 serve

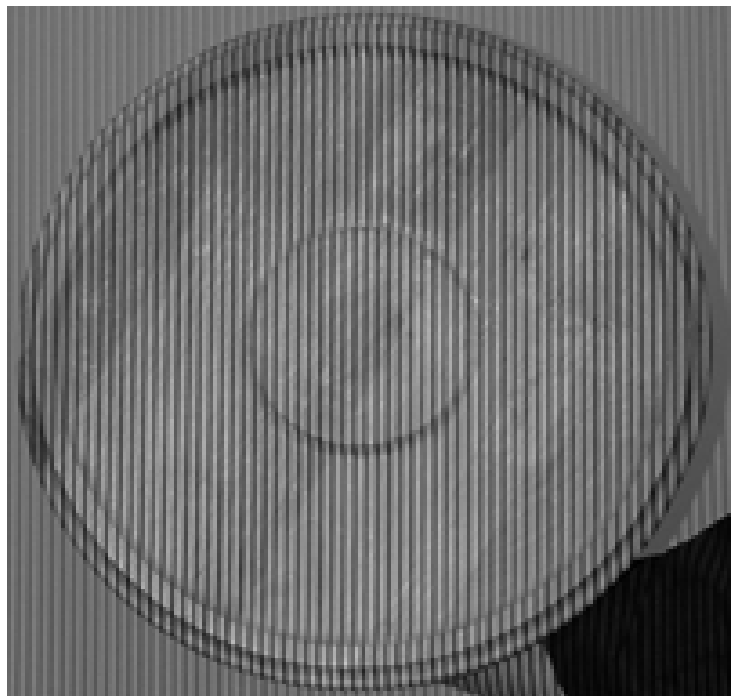

Figure 12. Deformed grating and the holder

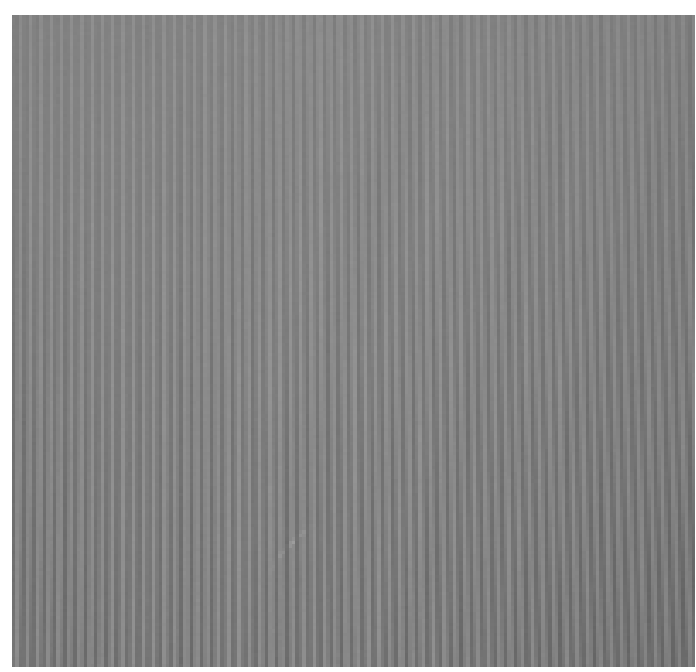

Figure 13. Sinusoidal grating image

as the reference signal for determining absolute phase values to be converted into a height distribution.

Figure 14 shows the recovered shape of the holder. Because the holder is not parallel with the reference plane, the blue part of the holder is close to the reference plane and the yellow part is far from the reference plane. The shape of the holder is recovered correctly.

Figures 15 and 16 show examples of the object profile, where the results were obtained by CMM and the FTP. The profile is obtained by cutting the holder along a diameter of the holder. 
Figure 15 was drawn by Microsoft excel and Figure 16 was drawn by Matlab. In Figure 15, the maximum distance of top and bottom of the holder is (72.2101$66.3307)=5.8794 \mathrm{~mm}$. In Figure 16, the maximum distance is $6.4 \mathrm{~mm}$. The error is $8 \%$. The shape of two profiles is same. Therefore this method is feasible.

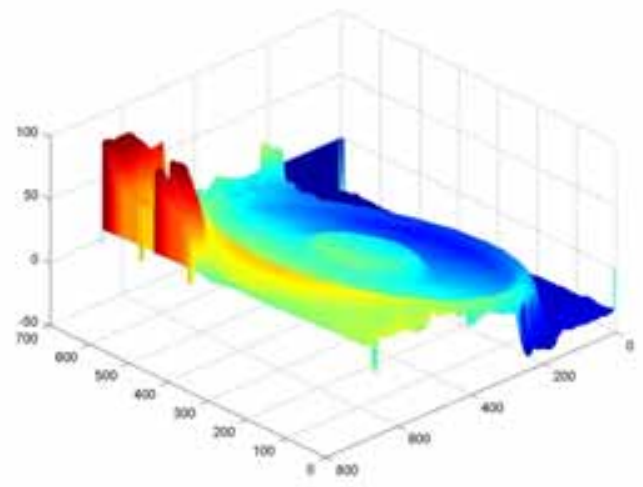

Figure 14. The recovered shape of the holder

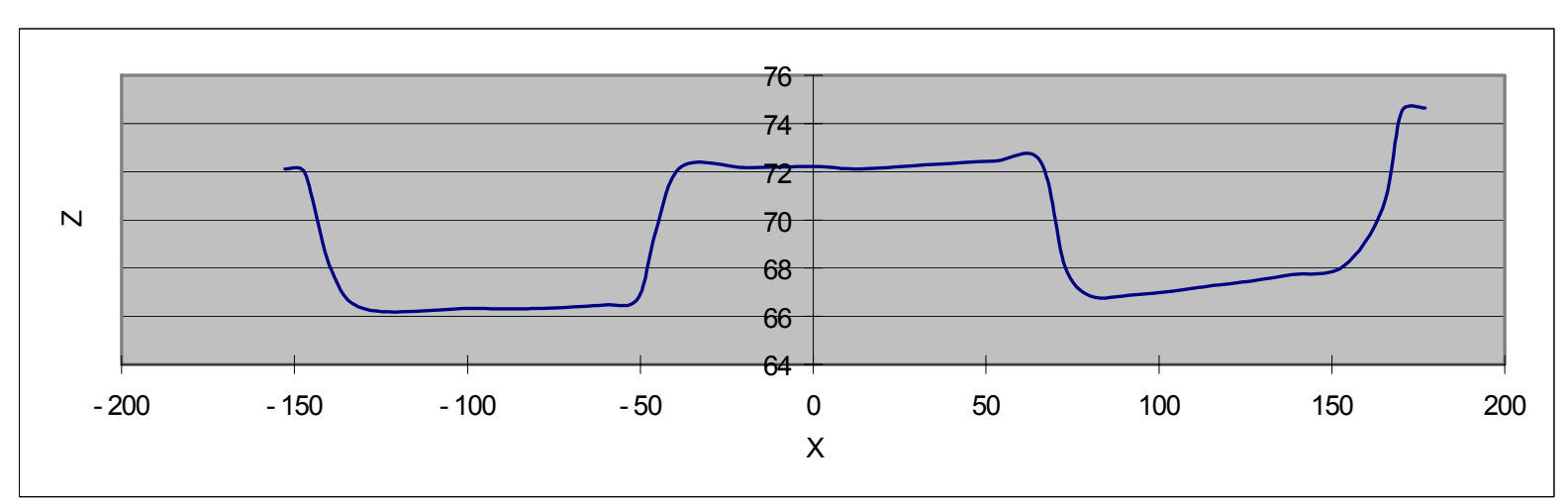

Figure 15. Profile of the holder that is measured by CMM

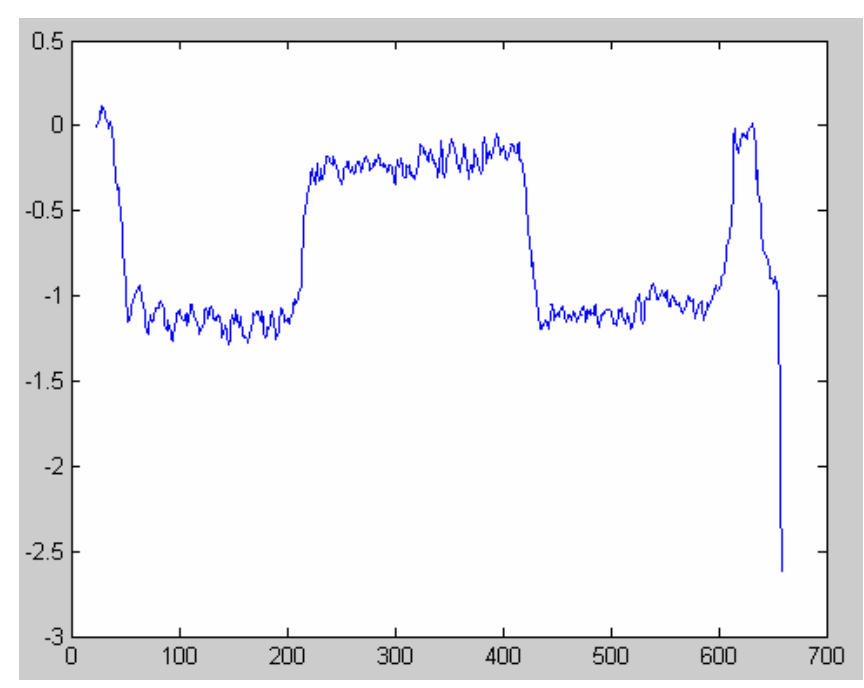

Figure 16. Profile of the holder that is obtained by FTP
The computing time of FTP method is approximately 140 minutes on an Intel ${ }^{\circledR}$ Pentium ${ }^{\circledR} 4$ microcomputer for a size of 1024 x 768 using Matlab. The reliabilityguided phase unwrap method takes a lot of computing time because the method searches for the whole image to find a local optimal modulation to unwrap phase. In practice, $\mathrm{C}++$ can be used to reduce the computing time. The reliability-guided phase unwrap method works effectively in Ref. [17].

\section{Conclusions}

This paper reports a FTP method to reconstruct a $3 \mathrm{D}$ object from its $2 \mathrm{D}$ image for the product modeling. A projector, a computer and measurement tools were used in this method, which provides a low-cost application. Experimental examples show the FTP method is a simple and feasible method. 
The approach described in this paper is still under development in the following directions:

(1) Improve the accuracy of the method.

(2) Decrease the computing time by $\mathrm{C}++$ coding.

\section{Acknowledgements}

This research is supported by Canadian NSERC Discovery Grants.

\section{Reference}

[1] Chang Woo Chu, Sungjoo Hwang and Soon Ki Jung, "Calibration-free Approach to 3D Reconstruction Using Light Stripe Projections on a Cube Frame", Third International Conference on 3-D Digital Imaging and Modeling, Vol.22, No.11, 2001, pp. 13-19.

[2] Q. Peng and M Loftus, "An Image-based Fast Threedimensional Modeling Method for Virtual Manufacturing", Journal of Engineering Manufacture, Vol 214, 2000, pp. 709-721.

[3] Jerry Isdale, “3D Scanner Technology Review”, http:// vr.isdale.com / 3Dscanners / 3Dscanner Review.html, 1998.

[4] Frank Chen, Gordon M. Brown and Mumin Song, "Overview of three-dimension Shape Measurement Using Optical Methods", Optical Engineering, Vol 39, 2000, pp. $10-22$.

[5] Stojanovic, R. S. Koubias, S. Stojanovic and E. Georgoudakis, "A Measuring Method for Laser-Based Profilometry and Its Application in Non-Destructive Testing and Quality Control", Proceedings of the 4th International Conference on Vibration Measurements by Laser Techniques -Advances\&Applications, http://www.apel.ee.upatras.gr/ the_lab/faculty/koubias_publications.htm.

[6] Roman Dovgard and Ronen Basri, "Statistical Symmetric Shape from Shading for 3D Structure Recovery of Faces", 8th European Conference on Computer Vision, LNCS 3022, 2004, pp. 99-113.

[7] Ruo Zhang, Ping-Sing Tsai, James Edwin Cryer, and Mubarak Shah, "Shape from Shading: A Survey", IEEE
Transactions on pattern analysis and machine intelligence, Vol.21, No. 8, 1999, pp.690-706.

[8] Zhengyou Zhang, "A Flexible New Technique for Camera Calibration", IEEE on Pattern Analysis and Machine Intelligence, Vol.22, No. 11, 2000, pp.1330-1334.

[9] Memon Qurban and Khan Sohaib, "Camera calibration and three-dimensional world reconstruction of stereo-vision using neural networks", International Journal of Systems Science, v 32, n 9, 2001, pp. 1155-1159.

[10] K.Achour and M.Benkhelif, "A new approach to 3D reconstruction without camera calibration", Pattern Recognition, Vol. 34, 2001, pp.2467-2476.

[11] Pollefeys M., Koch R., Vergauwen M. and Van Gool L., "Automated reconstruction of 3D scenes from sequences of images", Journal of Photogrammetry and Remote Sensing, v 55, n 4, Nov, 2000, p 251-267.

[12] Joaquim Salvimas, "An Approach to Coded Structured Light to Obtain Three Dimensional Information", Ph.D. Thesis, Unversity of Girona, 2001.

[13] C.Quan, X. Y. He, C.F. Wang, C.J. Tay, H.M. Shang, "Shape measurement of small objects using LCD fringe projection with phase shifting", Optics Communications, 2001, pp. 21-29.

[14] Wan-Song Li and Xian-Yu, Su, "Application of improved phase-measuring profilometry in nonconstant environmental light", Optical Engineering, Vol.40(3), 2001, pp. $478-485$

[15] Xianyu Su and Wenjing Chen, "Fourier transform profilometry: a review", Optics and lasers in Engineering, Vol. 35, 2001, pp. 263-284.

[16] Takeda, Mitsuo and Mutoh, Kazuhiro, "Fourier Transform Profilometry for the Automatic measurement of 3D Object Shapes", Applied Optics, Vol. 22, No. 4, 1983, pp.3977-3982.

[17] Xianyu Su and Wenjing Chen, "Reliability-guided phase unwrapping algorithm: a review", Optics and Lasers in Engineering, Vol. 42, 2004, pp 245-261.

[18] Giovanna Sansoni, Luca Biancardi, Umberto Minoni and Franco Docchio, "A Novel, Adaptive System for 3-D Optical Profilometry Using a Liquid Crystal Light Projector", IEEE Transactions on instrumentation and measurement, Vol 43, No. 4, 1994, pp558-566.

[19] "Camera Calibration Toolbox for Matlab", http://www.vision.caltech.edu/bouguetj/calib_doc/. 